\title{
Ewing Sarcoma of Soft Parts: About Three Cases and Literature Review
}

\author{
M. Ouali Idrissi, H. EL Hammaoui ${ }^{*}$, M. El Ghaidi, H. El Mortaji, B. Boutakioute, N. Cherif Idrissi Guanouni
}

Radiology department, Ar-razi hospital, CHU Med VI, Marrakech

DOI: $\underline{10.36347 / \text { sjmcr.2020.v08i01.004 }}$

| Received: 21.10.2019 | Accepted: 28.10.2019 | Published: 14.01.2020

*Corresponding author: H. EL Hammaoui

\section{Abstract}

Extraosseous Ewing sarcomas (EESs) are rare tumours originating from soft tissues. Imaging has an interest in the diagnosis of lesions, local extension and post-treatment follow-up. This paper reports tree cases of soft tissue Ewing's sarcoma. They all benefited from an MRI with the T1 and T2 weighted sequences in all three planes and FATSAT without and after gadolinium injection in all three planes. The guided echo biopsy was done for all the cases objectifying Ewing's sarcoma. They were treated by a multidisciplinary approach including surgery, chemotherapy and radiotherapy.

Keyboard: Ewing sarcoma, Soft parts, US, MRI.

Copyright @ 2020: This is an open-access article distributed under the terms of the Creative Commons Attribution license which permits unrestricted use, distribution, and reproduction in any medium for non-commercial use (NonCommercial, or CC-BY-NC) provided the original author and source are credited.

\section{INTRODUCTION}

ES is a rare and highly malignant small round cell tumor that primarily affects the skeletal system. In primary extra osseous ESs of soft tissue underlying bone involvement is not found. James Ewing described it in 1921 as a tumor arising from undifferentiated osseous mesenchymal cells; however, recent studies suggest that Ewing's tumor may be of neuroectodermal origin being derived from the primitive neural tissue [15]. Extraskeletal Ewing sarcoma is rare in comparison with Ewing sarcoma of bone [1]. The prevalence of extraskeletal Ewing sarcoma is generally accepted to be between $15 \%$ and $20 \%$ of that of Ewing sarcoma of bone and include the lower extremities (32\%), the paravertebral region, $(15 \%)$, the chest wall $(11 \%)$, and the retroperitoneum $(11 \%)[6,7]$.

This report describes 3 cases of extra osseous ES. It elucidates the importance of professional knowledge of the relevant aspects of ES.

\section{Case Reports}

\section{Case 1}

A 21year old women wold presented in the surgical outpatient department with history of a progressive swelling over the right shoulder region for the last year Swelling started as a small lump that increased in size during last five months. Swelling was not associated with fever, malaise and fatigue. There was no history of exposure to any carcinogenic agent or radiation. Past history was not significant.

The general examination of the patient was normal. Local examination revealed a globular swelling over the right shoulder region, measuring 60x90 mm, having firm to hard consistency. It was mobile with well-defined margins, shiny with multiple visible vessels and not attached to deeper structures. There was no neurovascular deficit distal to the tumour (figure 1).

Blood complete picture revealed mild anemia. Other blood tests including renal function test, and liver function test were within normal limits. The patient underwent sonography of the right shoulder region, which revealed a hypoechoic, round lesion in the soft tissues, approximately $60 \mathrm{~mm}$ by $76 \mathrm{~mm}$ in size, with internal vascular.

MRI of the shoulder region showed a voluminous necrosed supraclavicular tissue mass measuring 66x85x94mm, presented a low signal $\mathrm{T} 1$, Heterogenous high signal $\mathrm{T} 2$, and heteregenous with prominent enhancment T1 with Gd. However, there was no distant metastase (figure 2).

Ultrasound guided biopsy confirmed the diagnosis of ES (figure 3). The lesion was excised and histopathology confirmed the diagnosis of ES (figure 4). All resection margins were free of tumour. She was referred to oncologist for chemotherapy. 


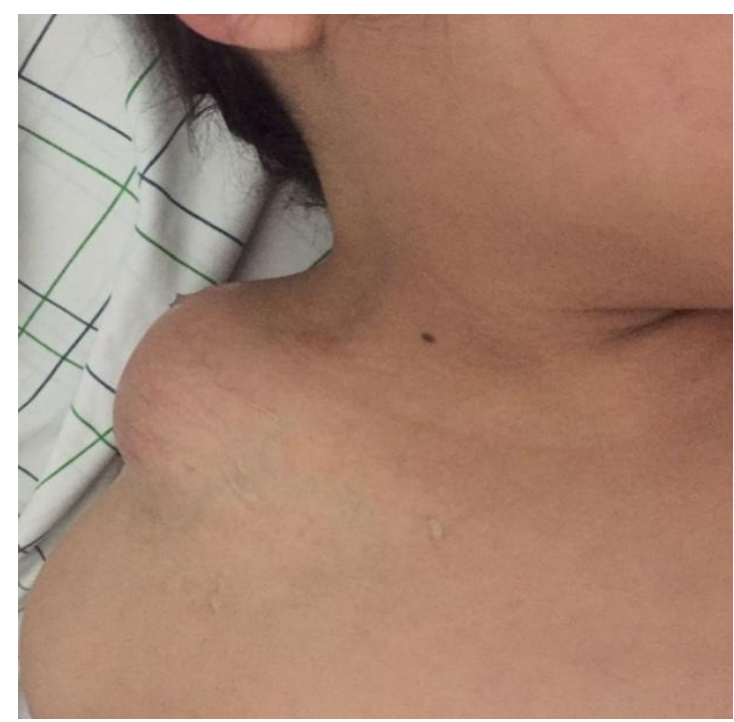

Fig-1: Mass at the right shouldar region
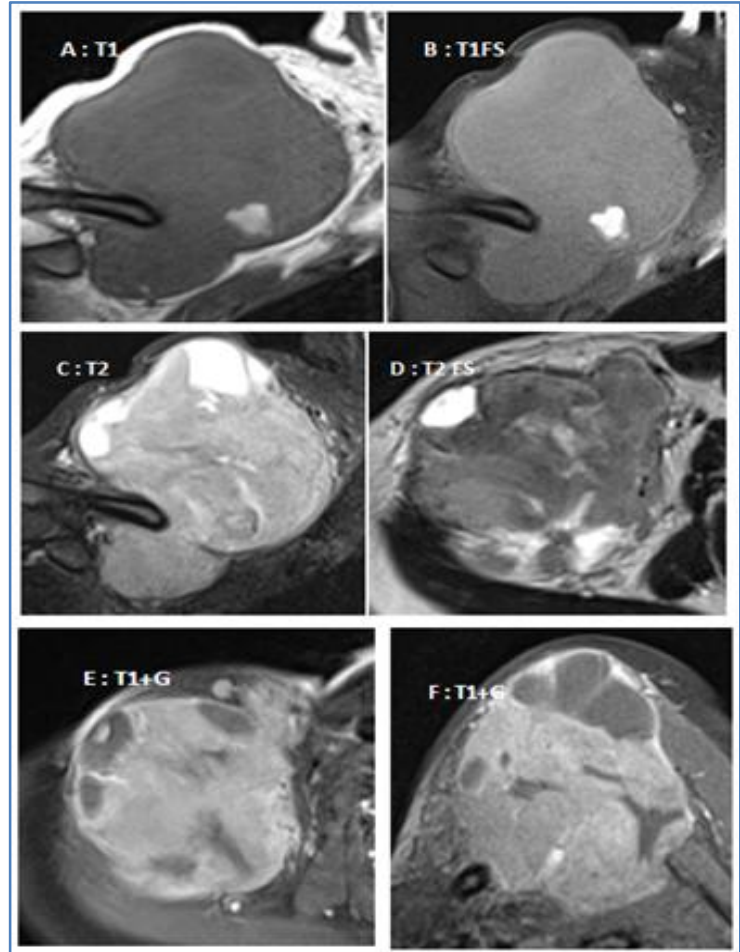

Fig-2: A, B, C, D, E, F: voluminous necrosed supraclavicular tissue mass, presented a low signal T1, Heterogenous high signal T2, and heteregenous with prominent enhancment $\mathrm{T} 1$ with $\mathrm{Gd}$

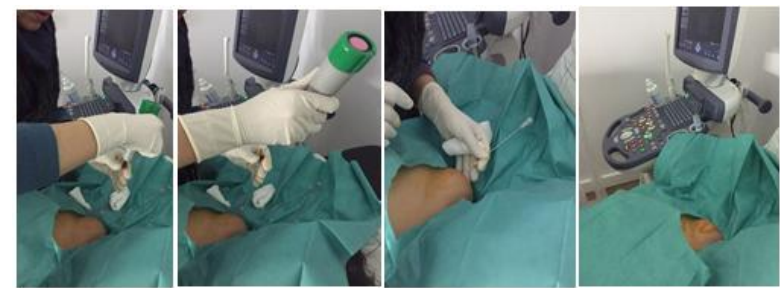

Fig-3: All steps of ultrasound guided biopsy

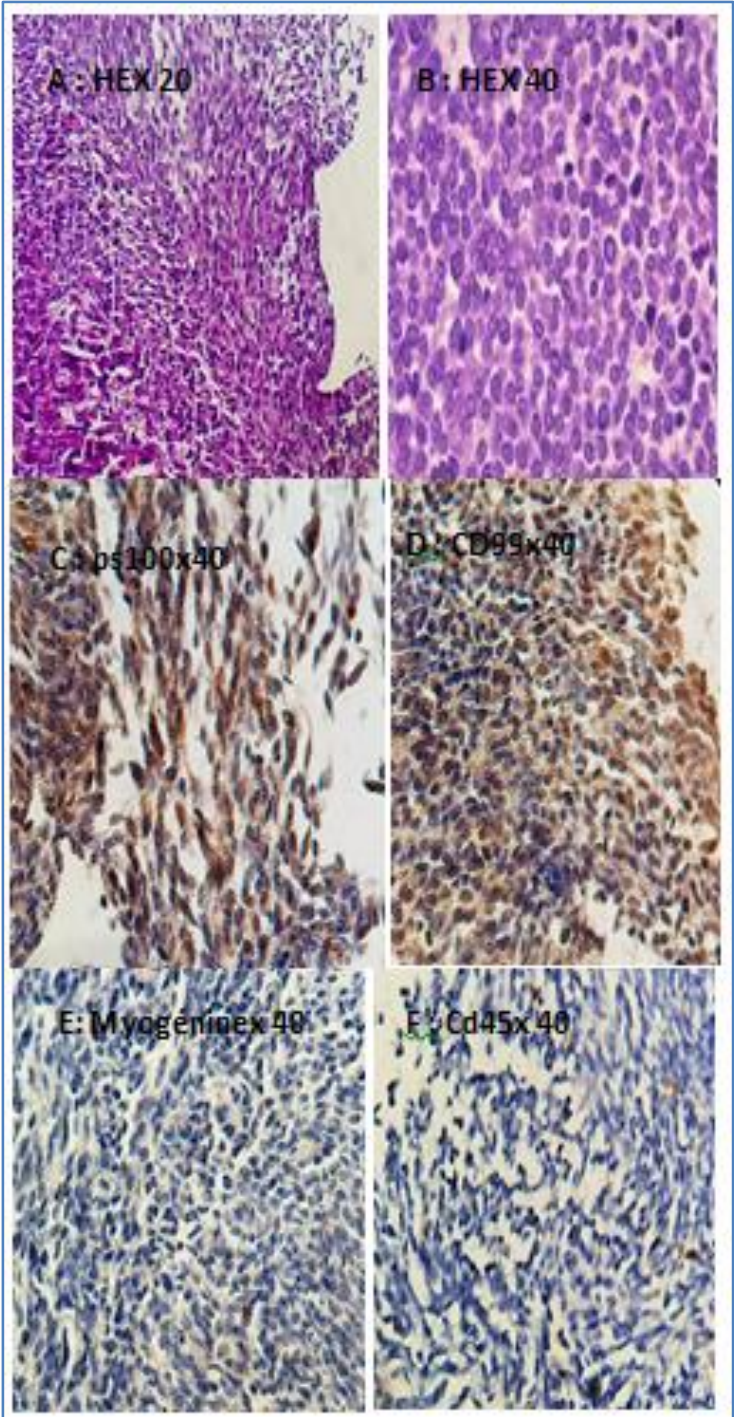

Fig-4: A, B: Sheets of small, round, uniform cells with scant clear cytoplasm, divided into irregular lobules by fibrous strands

- C, D: Positive staining of CD99 and ps 100.

- $\quad$ E, F: negative staining of myogenin and CD 45.

Case 2

A 14 years old child presented for a swelling of the right leg a increasing in volume. The clinical examination shows a huge mass firm, insensitive, fixed with respect to the superficial plane, without inflammatory signs opposite.

MRI objectived a large soft tissue tumor in the anterior compartment of the leg; It is intracompartmental under aponeurotic and develops at the expense of the anterior tibialis muscle without any realizable capsule. It's heterogeneously enhanced after contrast injection with hyposignal thin septum on all sequences related to haemorrhagic content (figure 5). The patient had a CT that did not show any pulmonary 
or other secondary. Ultrasound guided biopsy confirmed the diagnosis of ES.

The lesion was excised and histopathology confirmed the diagnosis of ES. All resection margins were free of tumour. He was referred to oncologist for chemotherapy.

MRI control showed a regression of the tumor of the soft parts of the right leg with persistence of a small residual lesion centered on the external cortex of Tibia measuring $8 \mathrm{~mm}$ thick spread over $5 \mathrm{~cm}$, presented a isosignal T1, high signal T2 and STIR, with prominent enhancment after gadolinium injection ( figure6).

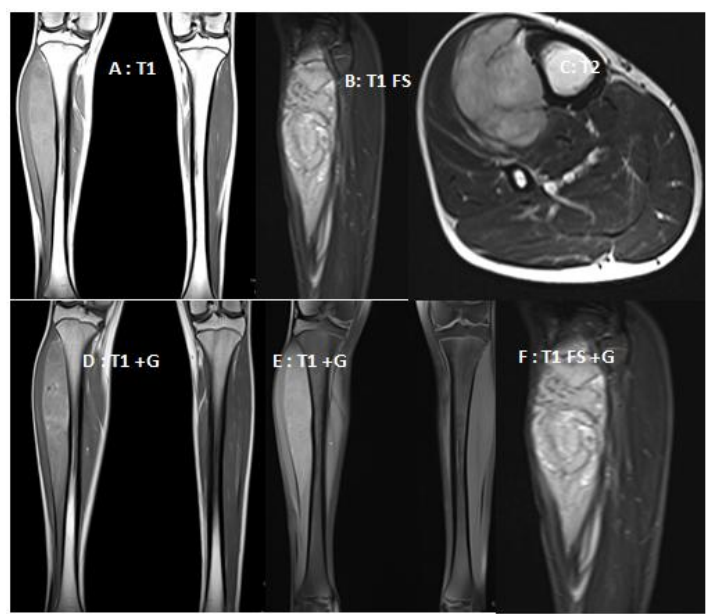

Fig-5: MRI objectived a large soft tissue tumor in the anterior compartment of the leg; It is intracompartmental under aponeurotic and develops at the expense of the anterior tibialis muscle without any realizable capsule. It's heterogeneously enhanced after contrast injection with hyposignal thin septum on all sequences related to haemorrhagic
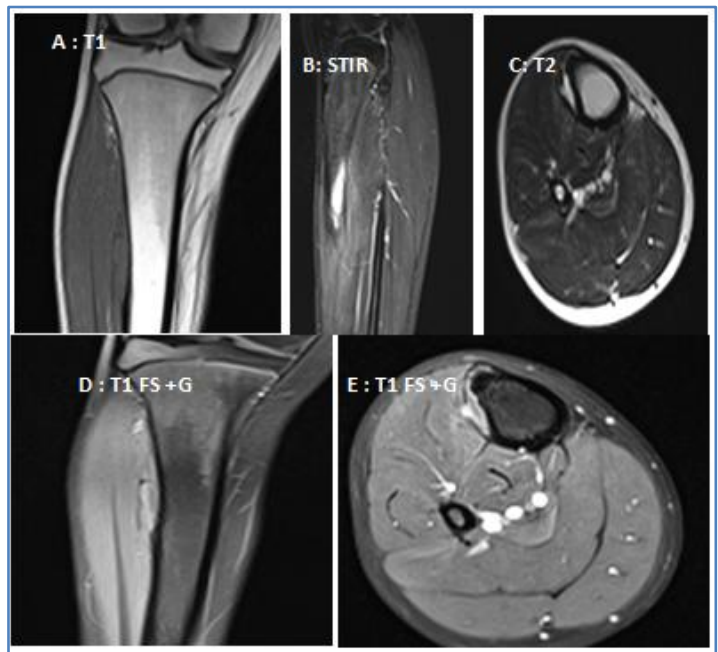

Figure 6: MRI control : regression of the tumor of the soft parts of the right leg with persistence of a small residual lesion centered on the external cortex of Tibia measuring $8 \mathrm{~mm}$ thick spread over $5 \mathrm{~cm}$, presented a isosignal T1, high signal T2 and STIR, with with prominent enhancment after gadolinium injection

\section{Case 3}

43 years old men presented for a swelling of the right leg a increasing in volume. The clinical examination shows a huge mass, with soft consistency, mobile and painful without inflammatory signs opposite. Special investigations included normal blood work and X-ray of the right shoulder.

X-ray of the right shoulder showed soft tissue swelling without any bony involvement (figure 7). MRI of the shoulder region showed a voluminous soft tissue mass, presented similar intensity to skeletal muscle on $\mathrm{T} 1$ and heterogenous high signal T2. It's heterogeneously enhanced after contrast injection with hyposignal thin septum on all sequences related to haemorrhagic (figure 8).

US-guided biopsy revealed a ewing sarcoma. The patient referred to oncologist for chemotherapy and was started on neoadjuvant chemotherapy.

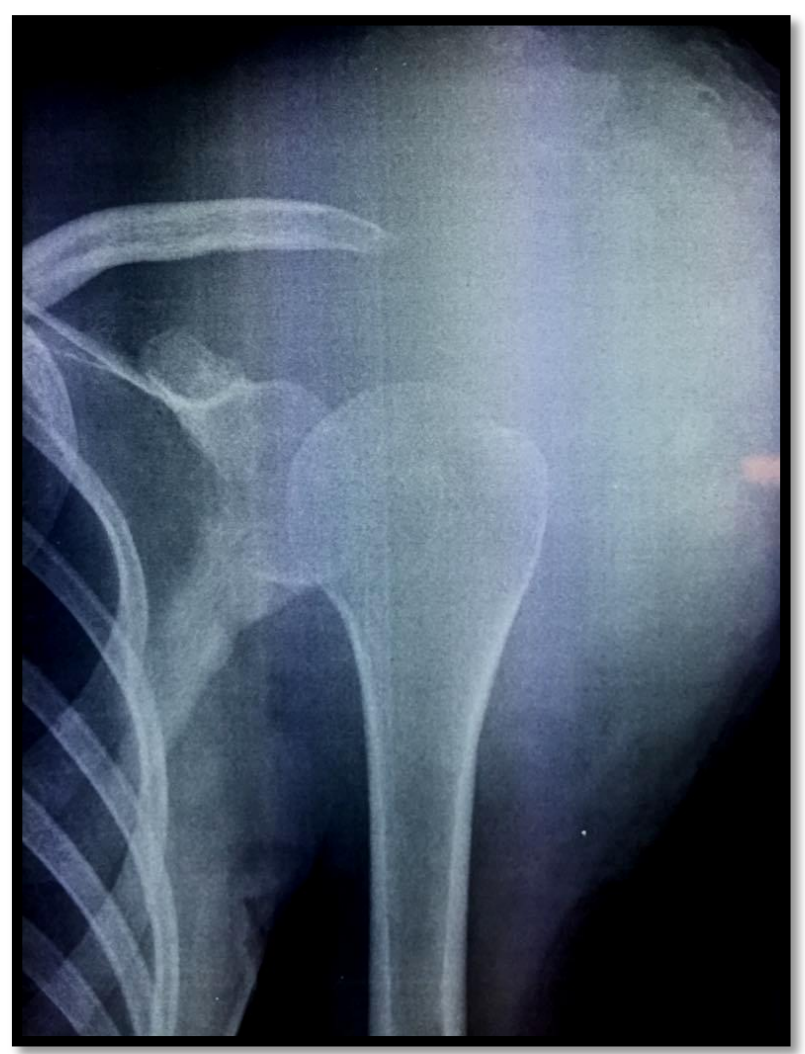

Fig-7: X-ray of the right shoulder showed soft tissue swelling without any bony involvement 


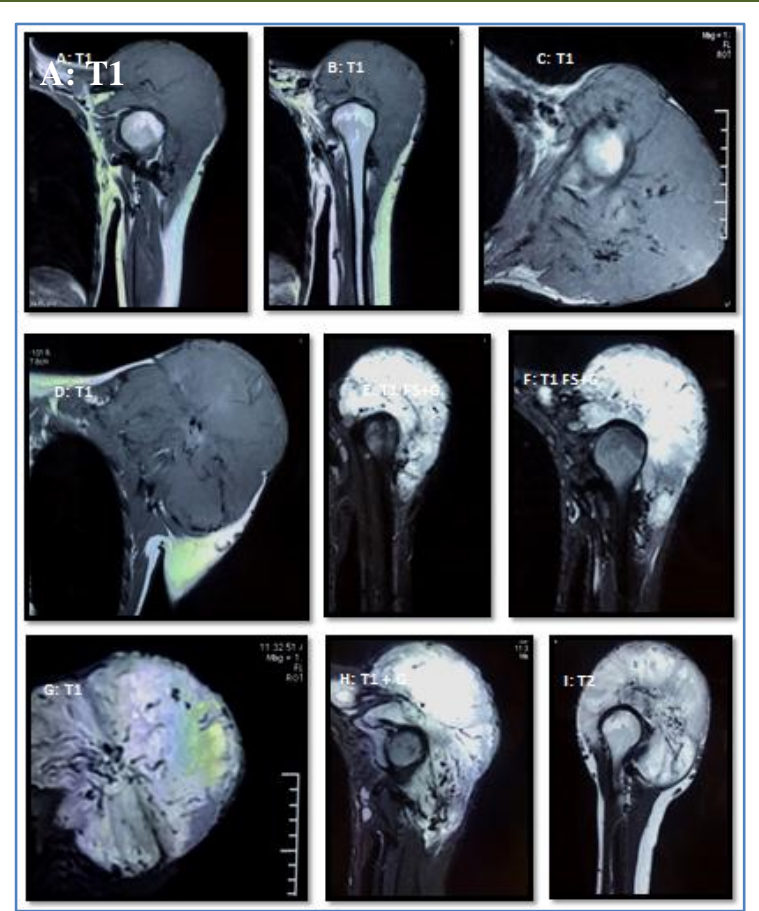

Fig-8: MRI of the shoulder region showed a voluminous soft tissue mass, presented similar intensity to skeletal muscle on T1.It's heterogeneously enhanced after contrast injection with hyposignal thin septum on all sequences related to haemorrhagic

\section{DISCUSSION}

Soft tissue ES is a rapidly growing, round-cell, malignant tumour which can reach $10 \mathrm{~cm}$ sizes by the time diagnosis is made [11]. Historically, Ewing sarcoma of soft tissue has included extraskeletal Ewing sarcoma and soft-tissue PNET[8, 9].

Extraskeletal Ewing sarcoma usually manifests in young patients, with $85 \%$ of cases detected between 20 months and 30 years of age [10]. However, historically and as with osseous lesions, in soft-tissue PNET the age range has been reported to be wider, from 1 month to 81 years $[8,9]$. The age range was 20 months to 63 years in Angervall and Enzinger's evaluation of 39 patients [11]. The median age of onset was 20 years in that study [11]. As with osseous lesions, extraskeletal Ewing sarcoma is rare in the black population [11].

The most frequent presenting symptom is a rapidly growing, solitary, superficial or deep mass with local pain $[5,12]$. The most commonly reported locations of extraskeletal Ewing sarcoma include the paravertebral region (32\%), lower extremities (26\%), chest wall $(18 \%)$, retroperitoneum $(11 \%)$, pelvis and hip (11\%), and upper extremities $(3 \%)$ [11, 13-16]. If the mass is seen to be paravertebral, the patient can present with symptoms of cord compression [11, 14]. Between $35 \%$ and $43 \%$ of adult patients have metastatic disease at presentation $[17,18]$. The most common site of metastasis is the lung [19]. ES may also present with systemic signs and symptoms such as weight loss and fever $[12,20]$.

Overall, imaging features of extraskeletal Ewing sarcoma are nonspecific. Initial diagnostic evaluation should begin with radiographs of the mass or region in question. At radiography, extraskeletal Ewing sarcoma may manifest as a large soft-tissue mass (50\% of cases) or demonstrate a normal appearance. Adjacent bone erosion, cortical thickening, osseous invasion, or aggressive periosteal reaction may also be present $(25 \%-42 \%$ of cases) $[15,21,22]$. Similarly, lesion calcification may be identified in up to $25 \%$ of cases $[15,21,22]$. Paravertebral masses can cause extrinsic bone erosion and secondary bone reaction in the adjacent vertebral body [23].

At ultrasonography (US), O'Keeffe and colleagues [24] reported that extraskeletal Ewing sarcoma lesions are most frequently hypoechoic. Anechoic areas may also be present, likely representing hemorrhage or necrosis [22, 24]. Increased Doppler blood flow is also present in extraskeletal Ewing sarcoma [22, 24].

Ultrasound criteria indicating a high suspicion of malignancy include increased size, irregular margins, heterogeneity and architectural distortion, whereas benign masses tend to be smaller, more homogeneous, well defined, superficially located and displacing rather than invading structures [25]. Ultrasonography can also be used to guide percutaneous needle biopsy [25].

The most effective modality for the detailed evaluation of osseous architecture is computerized tomography [25]. CT demonstrates a nonspecific softtissue mass, most commonly of similar attenuation to that of muscle (87\% of cases) [22, 24]. Low attenuation may also be seen, likely corresponding to areas of hemorrhage or necrosis $[22,24]$. Lesion margins are often poorly defined at CT (60\% of cases), which is likely a reflection of the more limited contrast resolution of $\mathrm{CT}$ in comparison with that of $\mathrm{MR}$ imaging [22]. Calcification is seen in $25 \%-30 \%$ of cases [22]. Osseous involvement of the bone surface with cortical erosion or periosteal reaction is seen in $40 \%$ of cases [22]. However, the medullary cavity retains its normal fatty marrow attenuation, a finding reflecting lack of involvement [22, 24].

MR imaging features are also nonspecific in evaluation of extraskeletal Ewing sarcoma. MR imaging demonstrates a soft-tissue mass with heterogeneous signal intensity $(91 \%)$ similar to that of skeletal muscle on T1-weighted images and intermediate to high signal intensity on T2-weighted images in $100 \%$ of cases [22]. High signal intensity on long TR images predominates in $64 \%$ of cases [22]. Intermediate-signal-intensity areas seen on long TR images are likely due to a high degree of cellularity, as 
in osseous lesions. Areas of hemorrhage appear as high signal intensity on all pulse sequences and are not uncommon; fluid levels may also be evident .Focal areas of necrosis with low signal intensity on T1weighted images and high signal intensity on T2weighted images are also frequent [22]. As in other soft-tissue masses, MR imaging is also useful for tumor staging and to evaluate the extent of involvement of surrounding structures [14].

Nowadays, bone scintigraphy and FDG PET can be also used to show increased radionuclide uptake [26]. Extraskeletal ES is confirmed by characteristic features on histological analysis, histochemistry, immunohistochemistry and electron microscopy $[5,12$, 27]. Differential diagnoses include other small, blue round cell tumours (SBRCTs) and other members of the Ewing family of tumours such as the primitive neuroectodermal tumour (PNET) [5, 12, 27].

Surgical resection, multi agent chemotherapy, and radio-therapy are the mainstay of treatment of ES $[5,12,27]$. The treatment plan should be individualized for each patient, which should be based on age, location, stage, size of the tumor and response to therapy $[5,12,27]$.

\section{CONCLUSION}

Ewing's sarcoma of soft parts is a rare mesenchymal tumor of poor prognosis. Receiving an early diagnosis increases the chance of survival. In the absence of clinical and radiological features, it seems necessary to include it in the differential diagnosis which includes all the primitive tumour of soft parts and to hypothesize this pathology when unusual localizations are found. Diagnostic imaging, especially MRI, allows comprehensive local staging and therapeutic follow- up.

\section{RÉFÉRENCE}

1. Naru T, Nawaz FH, Rizvi J. Juvenile Ewing's sarcoma presenting as a pelvic mass. J Coll Physicians Surg Pakistan. 2007; 17: 53- 4.

2. Maheshwari V, Siddiqui F, Adreena, Sherwani RK, Jain A, Alam K. Extraskeletal Ewing's sarcoma- A case report. Internet J Orthoped Surg. 2009; 14: 1.

3. Extraskeletal Ewing's sarcoma/primitive neuroectodermal tumor family. In: Enzinger and Weiss's Soft Tissue Tumors. 4th ed. St Louis: Mosby. 2001. pp. 1289- 91.

4. Túllio BM, Vieira FA, Rogério de FP, Vitorino CS, Mota LA. Ewing's sarcoma of the mandible in a young child. Braz Dent J. 2010; 21: 74-9.

5. Waqar SH, Zahid MA. Ewing's sarcoma in scapular region. APSP J Case Rep.2011; 2(3):22

6. Parham DM, Hijazi Y, Steinberg SM, Meyer WH, Horowitz M, Tzen CY, Wexler LH, Tsokos M. Neuroectodermal differentiation in Ewing's sarcoma family of tumors does not predict tumor behavior. Human pathology. 1999 Aug 1;30(8):911-8.

7. Javery O, Krajewski K, O'Regan K, Kis B, Giardino A, Jagannathan J, Ramaiya NH. A to Z of extraskeletal Ewing sarcoma family of tumors in adults: imaging features of primary disease, metastatic patterns, and treatment responses. American Journal of Roentgenology. 2011 Dec;197(6):W1015-22.

8. Dorfman HD. Ewing's sarcoma and related entities. Bone tumors. 1998.

9. Resnick D. Tumors and tumor-like lesions of bone: imaging and pathology of specific lesions. Diagnosis of bone and joint disorders. 1995.

10. Javery O, Krajewski K, O’Regan K, Kis B, Giardino A, Jagannathan J, Ramaiya NH. A to Z of extraskeletal Ewing sarcoma family of tumors in adults: imaging features of primary disease, metastatic patterns, and treatment responses. American Journal of Roentgenology. 2011 Dec;197(6):W1015-22.

11. Angervall L, Enzinger FM. Extraskeletal neoplasm resembling Ewing's sarcoma. Cancer 1975;36(1): 240-251

12. Askri A, Farhat LB, Ghariani B, Rabeh A, Dali N, Said W, Hendaoui L. Extraskeletal Ewing sarcoma of the abdominal wall. Cancer Imaging. 2008;8(1):156.

13. Soule EH, Newton Jr W, Moon TE, Tefft M. Extraskeletal Ewing's sarcoma. A preliminary review of 26 cases encountered in the inter group rhabdomyosarcoma study. Cancer. 1978 Jul;42(1):259-64.

14. Kennedy JG, Eustace S, Caulfield R, Fennelly DJ, Hurson B, O'Rourke KS. Extraskeletal Ewing's sarcoma: a case report and review of the literature. Spine. 2000;25(15):1996-1999.

15. Rose JS, Hermann G, Mendelson DS, Ambinder EP. Extraskeletal Ewing sarcoma with computed tomography Radiol. 1983;9(4): 234-237.

16. Harimaya K, Oda Y, Matsuda S, Tanaka K, Chuman H, Iwamoto Y. Primitive neuroectodermal tumor and extraskeletal Ewing sarcoma arising primarily around the spinal column: report of four cases and a review of the literature. Spine. 2003 Oct 1;28(19):E408-12.

17. Somarouthu BS, Shinagare AB, Rosenthal $\mathrm{MH}$, Tirumani H, Hornick JL, Ramaiya NH, Tirumani SH. Multimodality imaging features, metastatic pattern and clinical outcome in adult extraskeletal Ewing sarcoma: experience in 26 patients. The British journal of radiology. 2014 Jun 21;87(1038):20140123.

18. Tao H, Hu Y, Wang J. Asian Pacific Journal of Cancer Prevention. 2013;14 (5):

19. El Weshi A, Allam A, Ajarim D, Al Dayel F, Pant R, Bazarbashi S, Memon M. Extraskeletal Ewing's sarcoma family of tumours in adults: analysis of 57 
patients from a single institution. Clinical Oncology. 2010 Jun 1;22(5):374-81.

20. Cheung CC, Kandel RA, Bell RS, Mathews RE, Ghazarian MD. Extraskeletal Ewing sarcoma in a 77-year-old woman. Arch Pathol Lab Med. 2001; 125: 1358-60.

21. Angervall L, Enzinger FM. Extraskeletal neoplasm resembling Ewing's sarcoma. Cancer 1975;36(1): 240-251.

22. Robbin MR, Murphey MD, Jelinek JS, Temple HT. Imaging of soft tissue Ewing sarcoma and primitive neuroectodermal tumor [abstr]. Radiology. 1998;209(P):311.

23. Tefft M, Vawter GF, Mitus A. Paravertebral "round cell" tumors in children. Radiology. 1969;92(7): 1501-1509
24. O'Keeffe F, Lorigan JG, Wallace S. Radiological features of extraskeletal Ewing sarcoma. $\mathrm{Br} \mathrm{J}$ Radiol. 1990;63(750):456-460

25. Pallavi Aga, Ragini Singh, Anit Parihar, Umesh Parashari; Imaging Spectrum in Soft Tissue Sarcomas ; Indian J Surg Oncol. 2011 Dec; 2(4): 271-279.

26. Rose JS, Hermann G, Mendelson DS, Ambinder EP. Extraskeletal Ewing sarcoma with computed tomography correlation. Skeletal radiology. 1983 May 1;9(4):234-7.

27. Perouli E, Chrysikopoulos H, Vlachos A, Koskinas A, Batistatou A, Polyzoidis K. Imaging findings in paraspinal extra osseous Ewing sarcoma. JBRBTR: organe de la Societe royale belge de radiologie $(\mathrm{SRBR})=$ orgaan van de Koninklijke Belgische Vereniging voor Radiologie (KBVR). 2006;89(6):310-2. 